\title{
A Walk-In Screening of Dementia in the General Population in Taiwan
}

\author{
Chun-Hung Chen, ${ }^{1}$ Ling-Chun Wang, ${ }^{2}$ Tzu-Chiao Ma, ${ }^{2,3}$ and Yuan-Han Yang ${ }^{1,2,4,5}$ \\ ${ }^{1}$ Department of Neurology, Kaohsiung Medical University Hospital, Kaohsiung Medical University, Kaohsiung City 80708, Taiwan \\ ${ }^{2}$ Mentality Protection Center, Fo Guang Shan Compassion Foundation, Kaohsiung City 80050, Taiwan \\ ${ }^{3}$ Graduate Institute of Oral Health Sciences, Kaohsiung Medical University, Kaohsiung City 80708, Taiwan \\ ${ }^{4}$ Department of Neurology and Master's Program in Neurology, Faculty of Medicine, Kaohsiung Medical University, \\ Kaohsiung City 80708, Taiwan \\ ${ }^{5}$ Department of Neurology, Kaohsiung Municipal Ta-Tung Hospital, Kaohsiung Medical University, Kaohsiung City 80145, Taiwan
}

Correspondence should be addressed to Yuan-Han Yang; endless@kmu.edu.tw

Received 12 March 2014; Accepted 21 April 2014; Published 4 May 2014

Academic Editor: Huali Wang

Copyright (c) 2014 Chun-Hung Chen et al. This is an open access article distributed under the Creative Commons Attribution License, which permits unrestricted use, distribution, and reproduction in any medium, provided the original work is properly cited.

\begin{abstract}
Alzheimer's disease (AD) has increased in its prevalence due to the increasing aged population. Currently there is no updated data on the prevalence of dementia including its very mild stage in Taiwan. Under the extensive coverage of Mentality Protection Center (MPC), Fo Guang Shan, Taiwan, the volunteers of MPC have conducted the medicine-related services and the screening of dementia by AD8 (ascertainment of dementia 8) that can screen the dementia even at its very mild stage in general population in all Taiwan. From 2011 to 2013, in total, 2,171 participants, 368 in the northern, 549 in the central, 877 in the southern, and 377 in the eastern part, were recruited with the mean age being $66.9 \pm 10.2$ years old. The ratio of suspected dementia patients, AD8 score greater than or equal to 2, was $13.6 \%$ of all recruited participants with their mean AD8 score being $2.9 \pm 1.3$, mean age being $69.4 \pm$ 10.8 years old, and female predominance being $73.0 \%$. Although this is a screening study, it has extensive coverage of all Taiwan and the use of AD8 is capable of screening very mild dementia. A further study with a randomized sampling to examine the prevalence and incidence of dementia including its very mild stage is encouraged.
\end{abstract}

\section{Introduction}

Alzheimer's disease (AD) is the main cause of dementia in Taiwan [1]. At present, most available data and studies regarding the prevalence of $\mathrm{AD}$ in Taiwan were completed in the past two decades [2-7], and these studies were limited to certain areas, Kaohsiung city, Kinmen county, Taipei city, or Ilan city, which do not provide an overall understanding of dementia in Taiwan [1]. Moreover, the aged population has been increasing steadily in recent years. These published studies did not have sufficient data to reflect current status of the aged population. These published studies also did not sufficiently detect dementia at the very mild stage, and did not address the very mild stage of dementia. To date, little is known about the current status of dementia in the general population in Taiwan.
AD8 is a brief tool used to screen dementia, which was developed from Washington University in St. Louis [8], which is capable of screening very mild dementia in a general population and is used extensively in other countries, including Taiwan [9]. If the screening result of AD8 is 2, the individual would be considered having dementia including very mild dementia [9]. AD8 can be administered to an informant of demented patients and the patient himself with a similar discriminative rate to help with differentiating demented from nondemented subjects [10].

The Mentality Protection Center (MPC) is a nonprofit institution and was established under Compassion Foundation Fo Guang Shan in 2008 to provide medical and charitable services to the general population in the world through hundreds of branches of Fo Guang Shan. Working together with the branches of Fo Guang Shan, scattered throughout 
urban, suburban, and rural areas in each of the northern, central, southern, and eastern parts of Taiwan, the MPC has launched dementia-screening projects to assist the elderly population regarding screening for dementia by utilizing AD8 from 2011 to 2013. The screening project, including dementia, depression, and sleep disorder, was administrated to the voluntary walk-in aged population in these branches if they were aware of the medical service and the dementiascreening project. All of the screening results were recruited to the MPC headquarter for the statistical analyses.

\section{Material and Methods}

2.1. The Training of Interviewers. All of our interviewers were senior nurses or other medical-related specialists. Before their administration of AD8 to the elderly population, they had to receive a series of training courses with regard to dementia-related and medical-related topics and to practice the administration of AD8 with the general population by working with experienced interviewers and physicians with internships. All of the interviewers were volunteers of MPC and completed walk-in screenings in this project in Taiwan.

2.2. Walk-In Screening. There are 59 branches of the Mentality Protection Center in Taiwan. All of these branches are scattered throughout the northern, central, southern, and eastern parts of Taiwan and distributed in urban, suburban, and rural areas in each part of Taiwan. From March 1, 2010 to April 30, 2013, 53 walk-in screenings were conducted at these 59 branches of MPC. Every screening in a branch had to last for 1 day to provide the medical and charitable service to the general elderly population and the screening of dementia by AD8 [9], depression by Center for Epidemiologic StudiesDepression (CES-D) scale [11], and sleep disorder by Pittsburgh Sleep Quality Index (PSQI) [12]. For all 53 screenings, 7 screenings were conducted in the northern part, 19 in the central part, 24 in the southern part, and 3 in the eastern part of Taiwan to the general population if they were older than 50 years old. Every walk-in participant was voluntary to join and complete all screens.

2.3. Participants and Evaluations. All participants voluntarily joined the screening activity without any reward. CES-D, AD8, and PSQI were administrated to individuals after identifying age, gender, and living area. The individual was considered as suspected dementia if his/her AD8 total score was greater than 2 . Using these existed data for the statistical analyses was approved by the Kaohsiung Medical University Hospital Institutional Review Board (IRB). All the information related to the privacy or that can be identified was not recorded during the screening process.

2.4. Statistics. Data analysis was performed using SPSS (version 12.0.1 for Windows, SPSS Inc., Chicago, IL, USA). All statistical tests were two-tailed and an alpha of 0.05 was taken to indicate significance. Analysis of variance (ANOVA) was used to compare the difference of group mean for age and for AD8 total score among the four areas of Taiwan for all participants and for all suspected dementia individuals. $t$-test was used to compare the group mean of age and of AD8 total score between suspected dementia and nondemented individuals. Chi-square test was used to compare the proportion of each reported subitem of AD8 and gender between suspected dementia and nondementia subjects and among four areas, the northern, central, southern, and eastern parts of Taiwan.

\section{Results}

In total, 2,171 participants, 368 in the northern, 549 in the central, 877 in the southern, and 377 in the eastern part, were recruited with the mean age $66.9 \pm 10.2$ years old. The mean age was significantly different among four areas $(P<0.001)$. The age of participants from the eastern area were older, $70.0 \pm 10.0$ years old, than the other three areas (Table 1). Participants were predominantly female, and there were no significant differences with regard to gender proportion $(P=$ $0.485)$ although it was higher in the southern area $(71.5 \%)$, compared to others (Table 1). For the proportion of reported change in each subitem of $\mathrm{AD} 8$, there was a significant difference in AD8-2 (reduced interest in hobbies and other activities) among the 4 areas $(P=0.024)$, where the eastern area had a higher proportion (10.9\%). Similarly, there was a significant difference in AD8-3 (repeating of questions, stories, or statements $)(P<0.001)$ among the 4 areas, where the northern area had a higher proportion (13.6\%). In the general population, the top two frequently reported items were AD8-8 (consistent problems with thinking and/or memory) (13.3\%), followed by AD8-5 (forgetting correct month or year) (8.2\%) (Table 1$)$.

The mean age between suspected demented $(69.4 \pm 10.8)$ and nondemented subjects $(66.6 \pm 10.0)$ was significantly different $(P<0.001)$. The ratio of suspected demented subjects was not significantly different among the four areas $(P=0.854)$ as well as age $(P=0.162)$, gender $(0.383)$, and mean AD8 total score $(P=0.658)$ (Table 2$)$. In general, the ratio of suspected dementia patients was $13.6 \%$ of all recruited participants with their mean AD8 score being 2.9 \pm 1.3 , mean age being $69.4 \pm 10.8$ years old, and female predominance being $73.0 \%$ (Table 2 ).

Ratio of reported change for each subitem of AD8 and AD8 total score were significantly different between suspected demented and nondemented group $(P<0.001$ for each comparison of AD8 subitem and AD8 total score) (Table 3). In nondemented group, the AD8-8 (consistent problems with thinking and/or memory (6.4\%)), AD8-2 (reduced interest in hobbies/activities were frequently reported (3.1\%)), and AD8-5 (forgetting correct month or year $(3.0 \%))$ were the top 3 frequently reported change in AD8 subitems (Table 3). Alternatively, these proportions of AD8 subitems in nondemented subjects, in part, were similar to those in suspected demented subjects where the AD8-8 (consistent problems with thinking and/or memory (56.8\%)), AD8-7 (difficulty in remembering appointments (47.0\%)), and AD8-5 (forgetting correct month or year (40.9\%)) were frequently reported (Table 3 ). 
TABLE 1: Demographic characteristics of all recruited participants.

\begin{tabular}{|c|c|c|c|c|c|c|}
\hline & Northern & Central & Southern & Eastern & $P$ value & Total \\
\hline $\begin{array}{l}\text { Number } \\
(n, \%)\end{array}$ & $\begin{array}{c}368 \\
17.0 \%\end{array}$ & $\begin{array}{c}549 \\
25.3 \%\end{array}$ & $\begin{array}{c}877 \\
40.4 \%\end{array}$ & $\begin{array}{c}377 \\
17.4 \%\end{array}$ & & $\begin{array}{c}2171 \\
100 \%\end{array}$ \\
\hline $\begin{array}{l}\text { Age, years } \\
(\text { mean } \pm S D)\end{array}$ & $68.7 \pm 9.8$ & $65.0 \pm 10.0$ & $66.2 \pm 10.1$ & $70.0 \pm 10.0$ & $<0.001$ & $66.9 \pm 10.2$ \\
\hline $\begin{array}{l}\text { Female } \\
(n / N, \%)\end{array}$ & $\begin{array}{c}258 \\
70.1 \%\end{array}$ & $\begin{array}{c}383 \\
69.8 \%\end{array}$ & $\begin{array}{c}627 \\
71.5 \%\end{array}$ & $\begin{array}{c}253 \\
67.1 \%\end{array}$ & 0.485 & $\begin{array}{c}1521 \\
70.1 \%\end{array}$ \\
\hline $\begin{array}{l}\text { AD8 score } \\
(\text { mean } \pm S D)\end{array}$ & $0.6 \pm 1.0$ & $0.6 \pm 1.1$ & $0.6 \pm 1.1$ & $0.5 \pm 1.1$ & 0.715 & $0.6 \pm 1.1$ \\
\hline AD8 $-1^{*}$ & $15,4.1 \%$ & $13,2.4 \%$ & $27,3.1 \%$ & $6,1.6 \%$ & 0.184 & $61,2.8 \%$ \\
\hline AD8-2* & $18,4.9 \%$ & $41,7.5 \%$ & $68,7.8 \%$ & $41,10.9 \%$ & 0.024 & $168,7.7 \%$ \\
\hline AD8-3* & $50,13.6 \%$ & $39,7.1 \%$ & $52,5.9 \%$ & $27,7.2 \%$ & $<0.001$ & $168,7.7 \%$ \\
\hline $\mathrm{AD} 8-4^{*}$ & $8,9.6 \%$ & $26,4.7 \%$ & $39,4.4 \%$ & $10,2.7 \%$ & 0.098 & $83,3.8 \%$ \\
\hline $\mathrm{AD} 8-5^{*}$ & $25,6.8 \%$ & $52,9.5 \%$ & $76,8.7 \%$ & $25,6.6 \%$ & 0.304 & $178,8.2 \%$ \\
\hline $\mathrm{AD} 8-6^{*}$ & $16,4.3 \%$ & $26,4.7 \%$ & $53,6.0 \%$ & $19,5.0 \%$ & 0.565 & $114,5.3 \%$ \\
\hline AD8-7* & $29,7.9 \%$ & $38,6.9 \%$ & $81,9.2 \%$ & $24,6.4 \%$ & 0.253 & $172,7.9 \%$ \\
\hline AD8-8* & $45,12.2 \%$ & $71,12.9 \%$ & $127,14.5 \%$ & $45,11.9 \%$ & 0.551 & $288,13.3 \%$ \\
\hline
\end{tabular}

${ }^{*}$ Change in each AD8 subitem (number and percentage in that area).

AD8-1: problems with judgment; AD8-2: reduced interest in hobbies/activities; AD8-3: repeats questions, stories, or statements; AD8-4: trouble learning how to use a tool, appliance, or gadget; AD8-5: forgetting correct month or year; AD8-6: difficulty handling complicated financial affairs; AD8-7: difficulty remembering appointments; AD8-8: consistent problems with thinking and/or memory.

TABle 2: Demographic characteristic of participants suspected dementia*.

\begin{tabular}{lcccccc}
\hline & Northern $(N=368)$ & Central $(N=549)$ & Southern $(N=877)$ & Eastern $(N=377)$ & $P$ value & Total $(N=2171)$ \\
\hline$n, \%$ & $48,13.0 \%$ & $74,13.5 \%$ & $126,14.4 \%$ & $48,12.7 \%$ & 0.854 & $296,13.6 \%$ \\
Age, mean \pm SD & $70.4 \pm 9.9$ & $67.7 \pm 11.9$ & $68.9 \pm 9.5$ & $71.9 \pm 12.5$ & 0.162 & $69.4 \pm 10.8$ \\
Gender (Female $/ n, \%)$ & $36,75.0 \%$ & $55,74.3 \%$ & $98,77.8 \%$ & $27,56.3 \%$ & 0.383 & $216,73.0 \%$ \\
Mean AD8 score & $2.7 \pm 0.9$ & $3.0 \pm 1.3$ & $2.8 \pm 1.2$ & $2.9 \pm 1.6$ & 0.658 & $2.9 \pm 1.3$ \\
\hline
\end{tabular}

* Defined as AD8 total score $\geqq 2$.

TABLE 3: AD8 subitems in dementia and nondementia subjects.

\begin{tabular}{lccc}
\hline AD8 subitems & $\begin{array}{c}\text { Dementia } \\
(N=296)\end{array}$ & $\begin{array}{c}\text { Nondementia } \\
(N=1875)\end{array}$ & $P$ value \\
\hline Age & $69.4 \pm 10.8$ & $66.6 \pm 10.0$ & $<0.001$ \\
AD8-1 $(n, \%)$ & $40,13.5 \%$ & $21,1.1 \%$ & $<0.001$ \\
AD8-2 & $109,36.8 \%$ & $59,3.1 \%$ & $<0.001$ \\
AD8-3 & $114,38.5 \%$ & $54,2.9 \%$ & $<0.001$ \\
AD8-4 & $66,22.3 \%$ & $17,0.9 \%$ & $<0.001$ \\
AD8-5 & $121,40.9 \%$ & $57,3.0 \%$ & $<0.001$ \\
AD8-6 & $86,29.1 \%$ & $28,1.5 \%$ & $<0.001$ \\
AD8-7 & $139,47.0 \%$ & $33,1.8 \%$ & $<0.001$ \\
AD8-8 & $168,56.8 \%$ & $120,6.4 \%$ & $<0.001$ \\
Total score & $2.9 \pm 1.3$ & $0.2 \pm 0.4$ & $<0.001$ \\
\hline
\end{tabular}

AD8-1: problems with judgment; AD8-2: reduced interest in hobbies/ activities; AD8-3: repeats questions, stories, or statements; AD8-4: trouble learning how to use a tool, appliance, or gadget; AD8-5: forgetting correct month or year; AD8-6: difficulty handling complicated financial affairs; AD8-7: difficulty remembering appointments; AD8-8: consistent problems with thinking and/or memory.

\section{Discussion}

This study provides updated information on the current status of dementia and the frequently reported presentations for mild dementia of Taiwan. Although the study design did not utilize a randomized sampling method to examine the prevalence and incidence of dementia in Taiwan, it provided information of dementia coming from four areas, the northern, central, southern, and eastern parts of Taiwan, for an overall examination for dementia and reported the frequently reported complaints and symptoms of dementia, including very mild dementia.

Prevalence studies of dementia in Taiwan were mostly conducted 2 decades ago [2-7], which cannot reflect our current status. Importantly, those studies were also done sporadically and separately in a localized area of Taiwan with different study designs and various psychometrics so it was not easy to have this data put together to reflect the condition of dementia for all of Taiwan. Meanwhile, some of those studies were not capable of reflecting the status of very mild dementia, CDR0.5 [13], that was less reported and addressed in those studies [2-7].

Most of our recruited participants were female predominant with a mean age of $66.9 \pm 10.2$ years old. Such results might, in part, be related to a female predominance for people aged greater than 50 years old in Taiwan [14], and females might be more inclined to participate in this social facility as compared with male, especially with regard to religion.

For the significant discrepancies in reported change of the AD8 subitem, the proportion of AD8-3 (repeats questions, 
stories, or statements) was higher in the northern part (13.6\%), where participants mostly come from the greater Taipei area, the greatest population density, [15] that is the location of the capital and having a higher daily cost of living caused the residents to be more anxious and stressed. The anxiety and stress will cause people to have memory problems and to repeat questions, stories, or statements [16, 17]. AD8-2 (reduced interest in hobbies/activities) was found to be higher $(10.9 \%)$ in the eastern part of Taiwan, as compared to others. The participants recruited from eastern part mainly came from Taitung City and Taitung County which have the lowest population density [15] and less flourishing and prosperous economic status as compared to other areas in Taiwan. As such, the residents in those areas have few interests and hobbies and have quite unsatisfactory levels of living status, that may have influence the higher proportion of reported AD8-2.

These suspected demented patients, AD8 $\geq 2$, were also female predominant, which was similar to our previous publication that current demented patients were female predominant [18], and also in other publications that female were predominant in the prevalence of $\mathrm{AD}$ [19]. The mean age of these suspected demented subjects was younger than that of our previously reported mean age, $79.3 \pm 7.7$, for current AD patients in Taiwan [18]. The difference was mainly due to the recruited subjects in our previous study that were from a hospital-based study where a more advanced stage of demented patients are treated, especially in sites such as medical centers.

In the reported AD8 subitems, although each subitem of AD8 was shown to have a significant difference between suspected demented and nondemented subjects, there were differences in the top three reported AD8 subitems between them. The different items were the second high frequency of reported change of subitems, AD8-7 in suspected dementia and AD8-2 in nondementia subjects. AD8-7 (difficulty remembering appointments) was mainly from the result of a memory problem in which an individual cannot memorize recent events, the impairment of recent memory could be the initial presentation of very mild dementia, especially in $\mathrm{AD}$ $[20,21]$.

Individuals with depressive syndrome tended to lose interest and to have the memory problems $[19,22]$. The prevalence of depressive syndrome among community-dwelling elderly in Taiwan was $27.5 \%$ [23] and that would result in our nondemented subjects having reports of AD8-8, AD8-2, and AD8-5 subitems, with reduced interest and having memory problems.

Our study has several strengths worth highlighting. First, we used the same interviewers to administer the AD8 instrument in all screenings so that we would not have interrater differences with regard to the study results. Second we used the same instrument, AD8, to avoid the biases of different tools used in different screening sites, and since AD8 is capable of screening very mild dementia [8-10], it would be more sensitive as compared to other tools used in the past such as MMSE [2-4]. Third, we surveyed individuals in the northern, central, southern, and eastern parts of Taiwan to reflect the status of screening results for all of Taiwan, which should be more objective when compared to other published studies in limited areas [2-7] in order to more accurately reflect and report on the status of dementia in Taiwan. On the other hand, this study involves a number of limitations that need to be addressed. Firstly, this study did not involve randomized sampling of the general population and as a result our study cannot reflect the real prevalence and incidence of current dementia in Taiwan. Secondly, we only used AD8 without other laboratory data or imaging data to provide the clinical diagnosis of each suspected demented patient; however, AD8 has been validated as being reliable in terms of sensitivity and specificity in screening dementia [9] and this study focused on the screening, not the diagnosing of dementia. Third, the study was a walk-in screening. This means that only the individuals who could independently walk into or be brought into our branches for screening were recruited. If he/she was independent or could not be brought into our screening sites, he/she might not join the screening process so that our current study may therefore underestimate the real status of the prevalence of dementia.

This was a screening study performed with some limitations and strengths, but did involve extensive coverage of all Taiwan and the use of AD8 was capable of screening very mild dementia. A further study with a randomized sampling to examine the prevalence and incidence of dementia with its subtypes in Taiwan is encouraged.

\section{Conflict of Interests}

The authors declare that there is no conflict of interests regarding the publication of this paper.

\section{Acknowledgments}

We deeply appreciate all the work done by medical volunteers of Mentality Protection Center and funds from Compassion Foundation Fo Guang Shan.

\section{References}

[1] Y. T. Wu, H. Y. Lee, S. Norton et al., "Prevalence studies of dementia in mainland china, Hong Kong and taiwan: a systematic review and meta-analysis," PLoS ONE, vol. 8, no. 6, Article ID e66252, 2013.

[2] C. K. Liu, C. L. Lai, C. T. Tai, R.-T. Lin, Y. Y. Yen, and S. L. Howng, "Incidence and subtypes of dementia in southern Taiwan. Impact of socio-demographic factors," Neurology, vol. 50, no. 6, pp. 1572-1579, 1998.

[3] R.-T. Lin, C.-L. Lai, C.-T. Tai, C.-K. Liu, Y.-Y. Yen, and S.L. Howng, "Prevalence and subtypes of dementia in southern Taiwan: impact of age, sex, education, and urbanization," Journal of the Neurological Sciences, vol. 160, no. 1, pp. 67-75, 1998.

[4] C.-K. Liu, R.-T. Lin, Y.-F. Chen, C.-T. Tai, Y.-Y. Yen, and S.-L. Howng, "Prevalence of dementia in an urban area in Taiwan," Journal of the Formosan Medical Association, vol. 95, no. 10, pp. 762-768, 1996.

[5] H.-C. Liu, J.-L. Fuh, S.-J. Wang et al., "Prevalence and subtypes of dementia in a rural Chinese population," Alzheimer Disease and Associated Disorders, vol. 12, no. 3, pp. 127-134, 1998. 
[6] H.-C. Liu, S.-J. Wang, J.-L. Fuh et al., "The Kinmen Neurological Disorders Survey (KINDS): a study of a Chinese population," Neuroepidemiology, vol. 16, no. 2, pp. 60-68, 1997.

[7] H.-C. Liu, K.-N. Lin, E. L. Teng et al., "Prevalence and subtypes of dementia in Taiwan: a community survey of 5297 individuals," Journal of the American Geriatrics Society, vol. 43, no. 2, pp. 144-149, 1995.

[8] J. E. Galvin, C. M. Roe, K. K. Powlishta et al., "The AD8: a brief informant interview to detect dementia," Neurology, vol. 65, no. 4, pp. 559-564, 2005.

[9] Y.-H. Yang, J. E. Galvin, J. C. Morris, C.-L. Lai, M.-C. Chou, and C.-K. Liu, "Application of AD8 questionnaire to screen very mild dementia in Taiwanese," American Journal of Alzheimer's Disease and Other Dementias, vol. 26, no. 2, pp. 134-138, 2011.

[10] J. E. Galvin, C. M. Roe, C. Xiong, and J. C. Morris, "Validity and reliability of the AD8 informant interview in dementia," Neurology, vol. 67, no. 11, pp. 1942-1948, 2006.

[11] D. Shinar, C. R. Gross, and T. R. Price, "Screening for depression in stroke patients: the reliability and validity of the Center for Epidemiologic Studies Depression Scale," Stroke, vol. 17, no. 2, pp. 241-245, 1986.

[12] D. J. Buysse, C. F. Reynolds III, T. H. Monk, S. R. Berman, and D. J. Kupfer, "The Pittsburgh Sleep Quality Index: a new instrument for psychiatric practice and research," Psychiatry Research, vol. 28, no. 2, pp. 193-213, 1989.

[13] J. C. Morris, M. Storandt, J. P. Miller et al., "Mild cognitive impairment represents early-stage Alzheimer disease," Archives of Neurology, vol. 58, no. 3, pp. 397-405, 2001.

[14] Department of Civil Affair, Ministry of the Interior, Taiwan, http://sowf.moi.gov.tw/stat/month/elist.htm.

[15] Department of Civil Affair, Ministry of the Interior, Taiwan, http://www.moi.gov.tw/dca/02place_001.aspx.

[16] M. Owens, J. Stevenson, J. A. Hadwin, and R. Norgate, "When does anxiety help or hinder cognitive test performance? The role of working memorycapacity," British Journal of Psychology, vol. 105, no. 1, pp. 92-101, 2014.

[17] J. Chin, K. J. Oh, S. W. Seo, and D. L. Na, "Are depressive symptomatology and self-focused attention associated with subjective memory impairment in older adults?" International Psychogeriatrics, vol. 26, no. 4, pp. 573-580, 2014.

[18] W. F. Wang, P. Y. Chiu, Y. T. Lin, C. J. Hu, J. L. Fuh, and Y. H. Yang, "Registration of Alzheimer's disease in Taiwan: patient and informant," American Journal of Alzheimer's Disease and Other Dementias, vol. 29, no. 1, pp. 18-22, 2014.

[19] D. S. Davydow, K. Zivin, and K. M. Langa, "Hospitalization, depression and dementia in community-dwelling older Americans: findings from the National Health and Aging Trends study," General Hospital Psychiatry, vol. 36, no. 2, pp. 135-141, 2014.

[20] G. M. McKhann, D. S. Knopman, H. Chertkow et al., “The diagnosis of dementia due to Alzheimer's disease: recommendations from the National Institute on Aging-Alzheimer's Association workgroups on diagnostic guidelines for Alzheimer's disease," Alzheimer's and Dementia, vol. 7, no. 3, pp. 263-269, 2011.

[21] H. Jahn, "Memory loss in Alzheimer's disease," Dialogues in Clinical NeuroSciences, vol. 15, no. 4, pp. 445-454, 2013.

[22] K. D. Young, P. S. Bellgowan, J. Bodurka, and W. C. Drevets, "Behavioral and neurophysiological correlates of autobiographical memory deficits in patients with depression and individuals at high risk for depression," JAMA Psychiatry, vol. 70, no. 7, pp. 698-670, 2013.
[23] Y.-F. Tsai, S.-H. Yeh, and H.-H. Tsai, "Prevalence and risk factors for depressive symptoms among community-dwelling elders in Taiwan," International Journal of Geriatric Psychiatry, vol. 20, no. 11, pp. 1097-1102, 2005. 


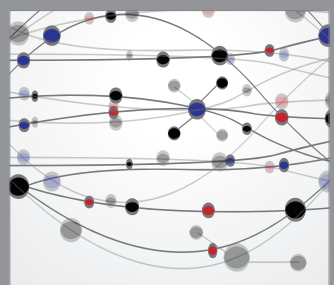

The Scientific World Journal
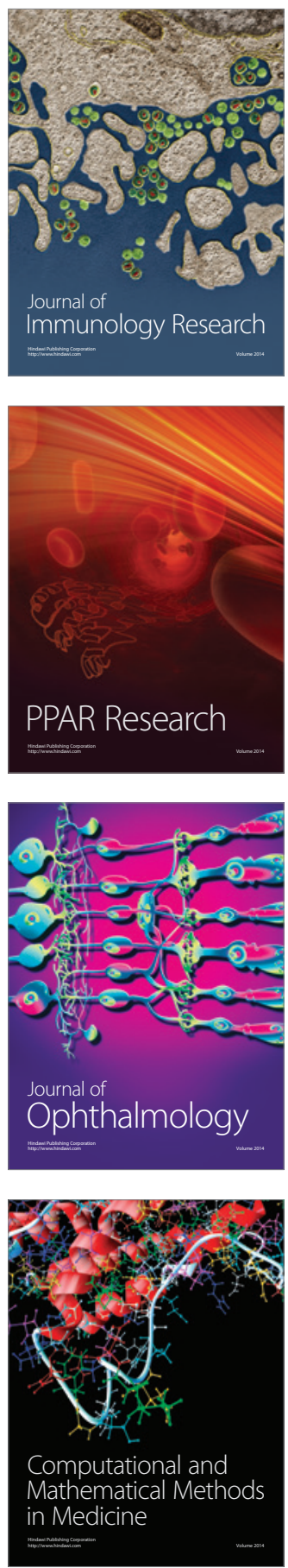

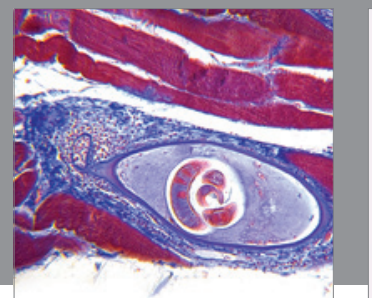

Gastroenterology

Research and Practice
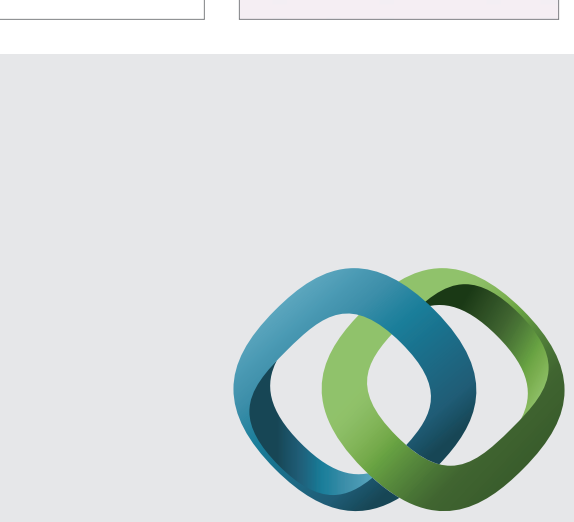

\section{Hindawi}

Submit your manuscripts at

http://www.hindawi.com
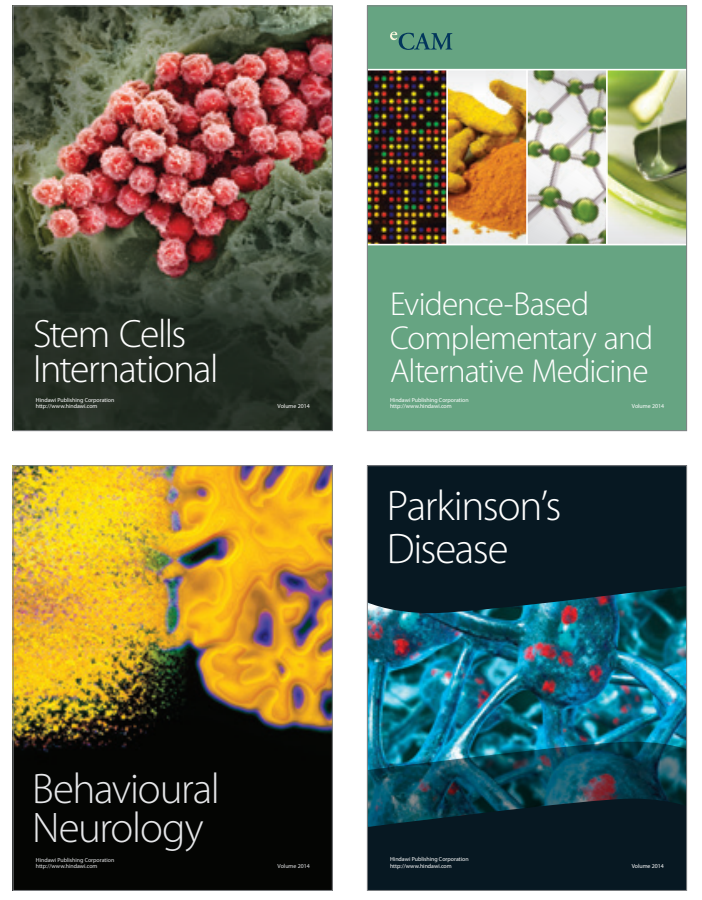
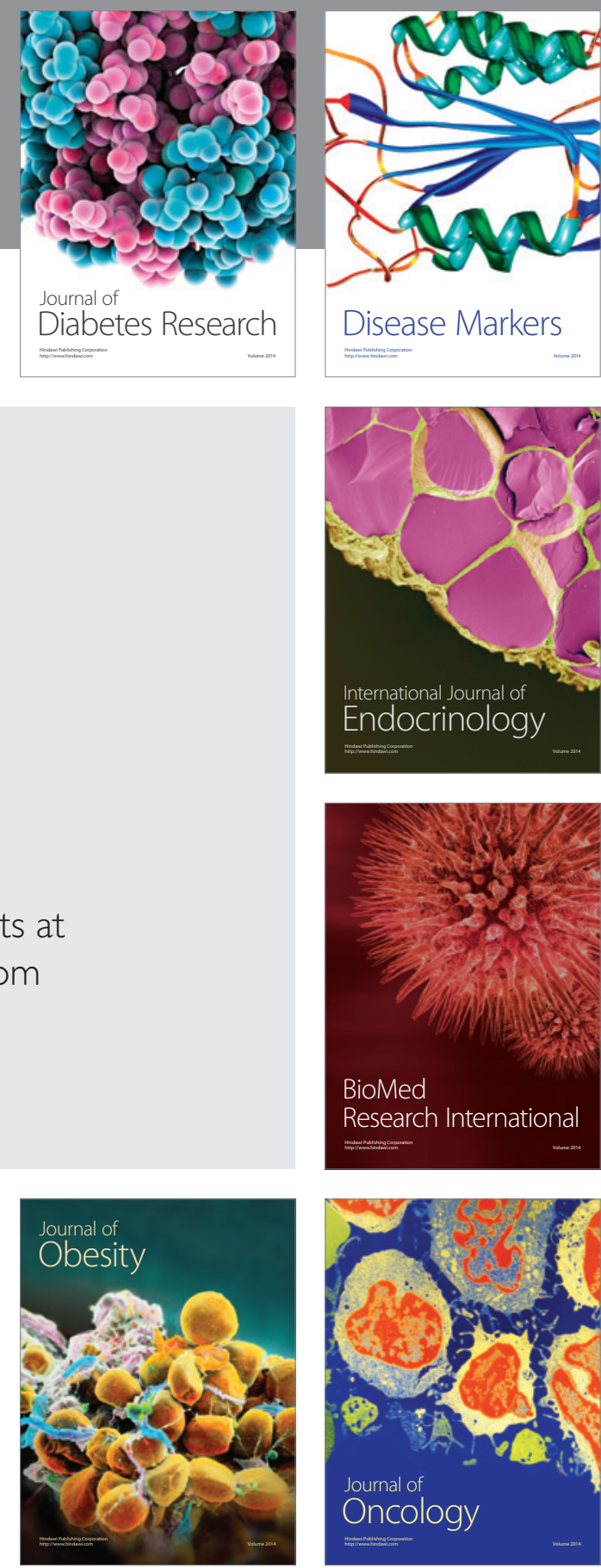

Disease Markers
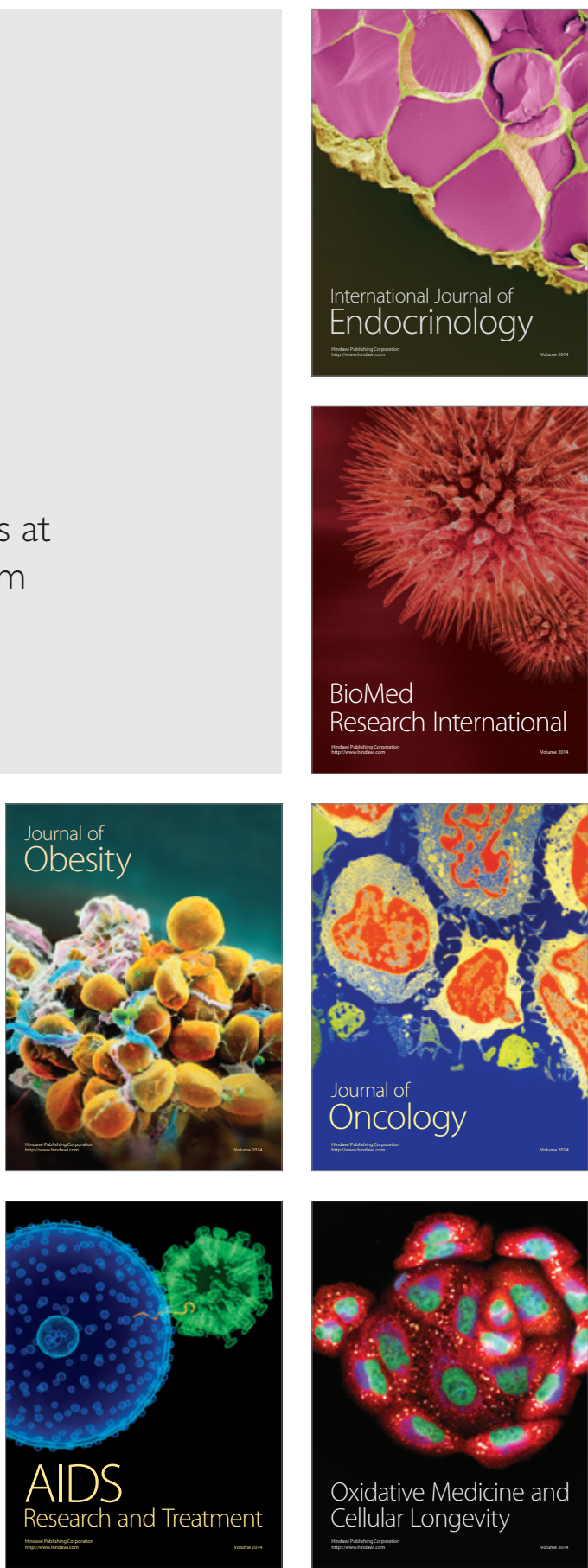